\title{
The Effect of miR-145-5p, DANCR and NRAS Expression Levels on the Survival Rate of Colorectal Cancer Patients
}

\author{
Fatemeh Bahreini1 $^{1 *}$, Massoud Saidijam ${ }^{2}$, Saeid Afshar ${ }^{2}$, Zahra Mousivand ${ }^{3}$, \\ Rezvan Najafi ${ }^{2}$
}

\begin{abstract}
Background: The prevalence of colorectal cancer $(\mathrm{CRC})$ has been increasing in the world. There are different factors for colorectal cancer, and changes in the levels of gene expression such as miR-145-5p, DANCR and NRAS can be one of the factors. Methods: The case-control study was performed on $40 \mathrm{CRC}$ specimens and 40 adjacent healthy tissues. Fresh tumor and tumor-free adjacent tissue samples were obtained from patients who underwent the surgical operation as a conventional treatment procedure. After tumor resection, the specimens were immediately frozen in liquid nitrogen and stored at $-80^{\circ} \mathrm{C}$ until further investigation. Cox regression was used to get the hazard ratios. Results: The mean \pm $\mathrm{SD}$ age of the patients was $62.45 \pm 12.89$ years. Of these, $62.5 \%$ were males. The risk of death from CRC in patients with low miR-145-5p levels is about 10 times higher than the high expression levels $(\mathrm{HR}=10.759, \mathrm{P}=0.009)$. High expression levels of NRAS can increase the risk of $\mathrm{CRC}$ death up to 4 times $(\mathrm{HR}=4.12, \mathrm{P}=0.045)$. The study did not show a relationship between DANCR expression levels and death risk from $\mathrm{CRC}(\mathrm{HR}=1.582, \mathrm{P}=0.439)$. Conclusion: These expression levels revealed that miRNA-145-5p and NRAS can be utilized as diagnostic biomarkers in colorectal cancer death. This may also introduce the microRNAs as colorectal cancer therapeutic targets.
\end{abstract}

Keywords: Colorectal neoplasm- gene expression- biomarker- survival rate- death

Asian Pac J Cancer Prev, 22 (12), 4043-4049

\section{Introduction}

The prevalence of colorectal cancer has been increasing in recent decades (Taheri Soodejani et al., 2019). Colorectal cancer is the second and third types of cancer diagnosed among men and women, respectively (Lee et al., 2018).

The prevalence of colorectal cancer varies around the world, but researchers have shown that the prevalence of colorectal cancer in developed countries is three times higher than that in developing countries (Rawla et al., 2019). The prevalence of colorectal cancer in Iran is lower than that in developed countries, but it is increasing (Maajani et al., 2019). The study on the burden of diseases in 2017 showed that the age-adjusted incidence of colorectal cancer was 23.2 per 100,000 person-years, and it has an increase of about $9.5 \%$ compared to 1990 (GBD 2017 Colorectal Cancer Collaborators, 2019).

According to the results of the study 2017, the age-adjusted incidence of colorectal cancer in Iran between 1990 and 2017 increased about 3.63\%. Statistics show that the incidence and mortality of colorectal cancer are increasing in low- and middle-income countries, while in developed countries it is stable or declining (Aguiar
Junior et al., 2020).

Colorectal cancer imposes directly or indirectly a large socio-economic burden on a community, and the survival rate of colorectal cancer can be due to health services in patient management. Therefore, it is important to identify the factors affecting colorectal cancer in any society (Lee et al., 2018).

Genetic factors in addition to environmental and demographic factors, can play a role in colorectal cancer. Some studies have shown that the expression of mir-145$5 \mathrm{p}$ can affect the incidence of colorectal cancer (Cheng et al., 2020; Vardini et al., 2017). Some studies have also shown that expressing mir-145-5p can increase the risk of colorectal cancer by 1.66 times (Xu et al., 2019). Therefore, this study was performed to investigate the relationship between miR-145-5p, DANCR and NRAS expression levels and survival rate of patients with colorectal cancer.

\section{Materials and Methods}

\section{Data}

This study was a case-control study performed on 40 colorectal cancer specimens and 40 adjacent healthy

${ }^{1}$ Department of Molecular Medicine and Genetics, Faculty of Medicine, Hamadan University of Medical Sciences, Hamadan, Iran. ${ }^{2}$ Research Center for Molecular Medicine, Hamadan University of Medical Sciences, Hamadan, Iran. ${ }^{3}$ Zabul University of Medical Sciences, Zabol, Iran.*For Correspondence: f.bahreini2824@gmail.com 
tissues provided by the Iran National Tumor Bank from 2013 to 2018. Exclusion criteria include patients who received any treatment, history of other cancers, chemotherapy, radiotherapy, diabetes mellitus, renal failure, and hypertension. The study was approved by the Ethics Committee of Hamadan University of Medical Sciences (Ethical code: IR.UMSHA.REC.1397.310). Colorectal tumor and tumor-free adjacent tissue samples were obtained from patients who underwent the surgical operation as a conventional treatment procedure. After tumor resection, the specimens were immediately frozen in liquid nitrogen and stored at $-80{ }^{\circ} \mathrm{C}$ until further investigation. For survival analysis, death was considered as the final condition of the samples over a period of 36 months.

\section{Real-time PCR}

Total RNA was isolated separately from tumor and normal tissue samples using the RNX-Plus solution according to the manufacturer's instruction (Sinaclone, Iran). Thereafter, miRNAs were reversely transcribed to cDNA by miRNA cDNA synthesis kit (Exir gene, Iran) according to the manufacturers' protocol. Both LncRNAs and mRNAs were reversely transcribed to cDNA by First Strand cDNA Synthesis Kit (Fermentas, USA), based on the manufacturers' protocol. Quantitative Real-Time PCR (qRT-PCR) reaction for the analysis of miRNA, LncRNAs, and mRNA expression was performed using SYBR ${ }^{\circledR}$ Premix ExTaq ${ }^{\text {TM }}$ II Kit (Takara, Japan), and Roche LightCycler® 96 system (Roche, Germany) according to the manufacturers' instructions. The primer pairs for genes LncRNAs and mRNAs were designed by allele ID 6 and their specificity was evaluated by NCBI primer blast (Table 1). Primers of has-miR-145-5p and u6 were purchased from Exir Gene Inc.

The level of $\beta$-actin (Gene ID: 60) and u6 (Gene ID: 26827) genes were used as a control. The results were normalized against Reference genes ( $\mathrm{u} 6$ and $\beta$-catenin). Relative expression of DANCR (Gene ID:57291), miR145-5p (Gene ID: 406937), and NRAS (Gene ID: 4893) was calculated using the $2^{-\Delta \Delta \mathrm{CT}}$ method

\section{Statistical analysis}

In this study, qualitative variables were described by number and percentage; quantitative variables were described as a mean \pm standard deviation. Independent t-test and one-way analysis of variance were used to assess the relationship between the expression levels of miR-145$5 \mathrm{p}$, DANCR and NRAS with demographic and clinical characteristics of patients separately. The expression levels of miR-145-5p, DANCR and NRAS were divided into two categories of low- and high- expression based on the median value. Then, we compared the survival rate of patients using Kaplan-Meier survival curve and Log-Rank test. Cox univariate/ multivariate regression was used to obtain the hazard ratios. In this study, a significant level was considered to be less than 5\%. Statistical analysis was performed using SPSS version 16 for windows (SPSS Inc., Chicago, IL).

\section{Results}

In the study, 40 colorectal tumor samples and 40 samples of healthy tissue adjacent to the same patients were selected as the control group. The mean $( \pm \mathrm{SD})$ age of the samples was 62.45 (12.89) with a range of 35-83 years. In this study, $37.5 \%$ of patients were females and $62.5 \%$ were males. The expression levels of miR-145-5p were significantly different in patients with body mass index (BMI) 25 and above compared to subjects who had a BMI less than $25 \mathrm{~kg} / \mathrm{m}^{\wedge} 2(\mathrm{p}=0.013)$. Also, the expression levels of miR-145-5p were significantly different in various stages of the cancer $(p=0.025)$. The results showed that there was no significant relationship between the expression levels of miR-145-5p and other variables in Table 2 .

The finding showed that the levels of DANCR expression were only significantly related to the age $(p=0.026)$ so that the difference between the levels of the expression in patients over 60 years and younger was 1.5 (Table 2). In this study, there was no difference between the mean expression levels of DANCR in the categories of other demographic and clinical variables of patients (Table 2). The mean expression of NRAS in smokers was lower than that of non-smokers $(p=0.006)$. Also, the mean expression of NRAS was not significantly different in various categories of the other variables (Table 2 ).

Table 3 shows the results of Cox univariate and multivariate regression analysis. The Cox multivariate regression analysis showed that by adjusting the effect of demographic and clinical variables, the expression levels of miR-145-5p and NRAS are effective on the hazard ratio of colorectal cancer. The risk of death from colorectal cancer in patients with low miR-145-5p levels is about 10 times higher than the high expression levels $(\mathrm{HR}=10.759, \mathrm{P}=0.009)$. In addition, high expression levels of NRAS can increase the risk of colorectal cancer death up to 4 times compared to low expression levels $(\mathrm{HR}=4.12, \mathrm{P}=0.045)$.

The results did not show a relationship between DANCR expression levels and death risk of colorectal cancer $(\mathrm{HR}=1.582, \mathrm{P}=0.439)$. Cox multivariate regression analysis showed that the risk of death for patients in stage III+IV was almost twice as high as that for stage I $(\mathrm{HR}=2.274, \mathrm{P}=0.039)$.

The mean survival time of patients with low miR-145-5p expression levels $(23.80 \pm 2.69,95 \% \mathrm{CI}=18.52-29.08$ months) was less than high expression levels (33.45 \pm 2.11 ,

Table 1. Primer Sequences for qRT-PCR

\begin{tabular}{lcccc}
\hline Gene & Gene ID & Annealing & Sense strand & Antisense strand \\
\hline$D A N C R$ & 57291 & 56 & CCTTGAGCTCCAGGAGTTCGTCT & GCTTGTGCCTGTAGTTGTCAACCT \\
$N R A S$ & 4893 & 55 & ATGACTGAGTACAAACTGGTGGT & CATGTATTGGTCTCTCATGGCAC \\
$\beta$-actenin & 60 & 53 & AAGATCAAGATCATTGCT & TAACGCAACTAAGTCATA \\
\hline
\end{tabular}



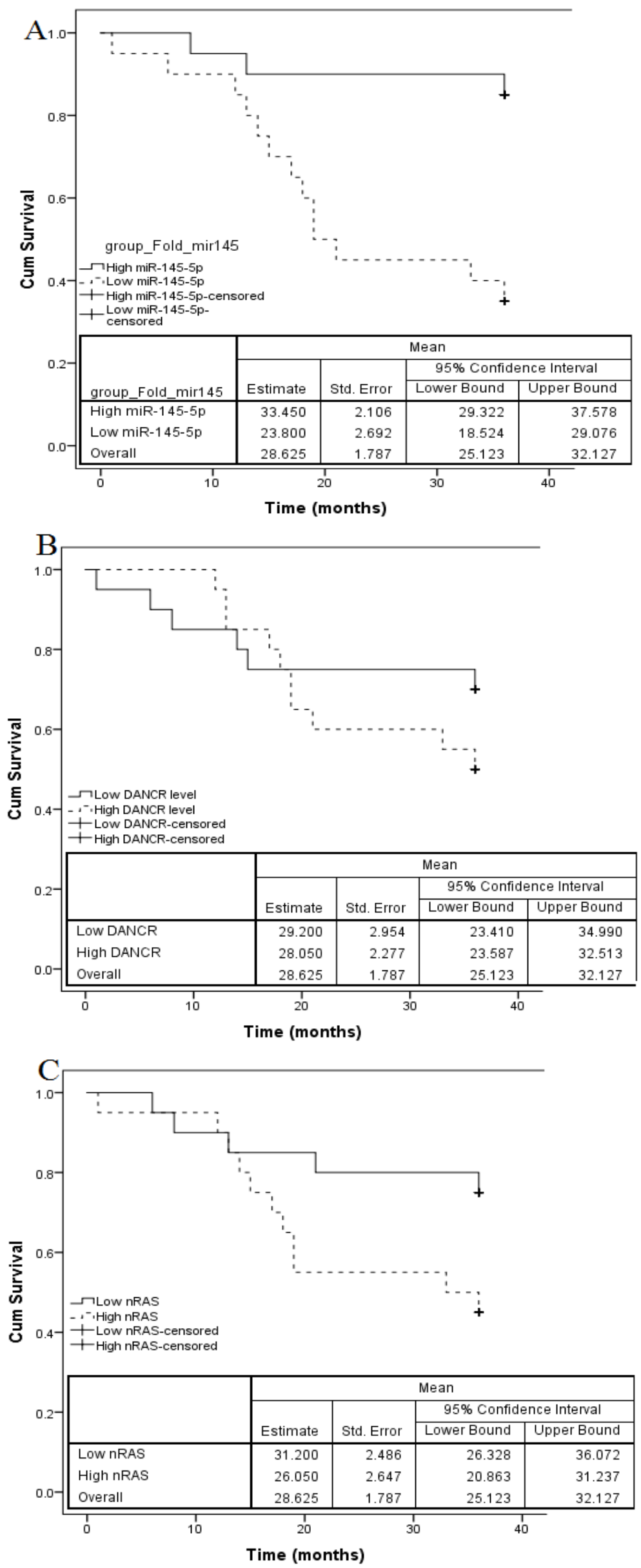

Figure 1. Kaplan-Meier Curve Shows the Survival Trend at Different Levels of miR-145-5p (A), DANCR (B) and NRAS (C) Expression. Log-rank test was used to compare the levels. 


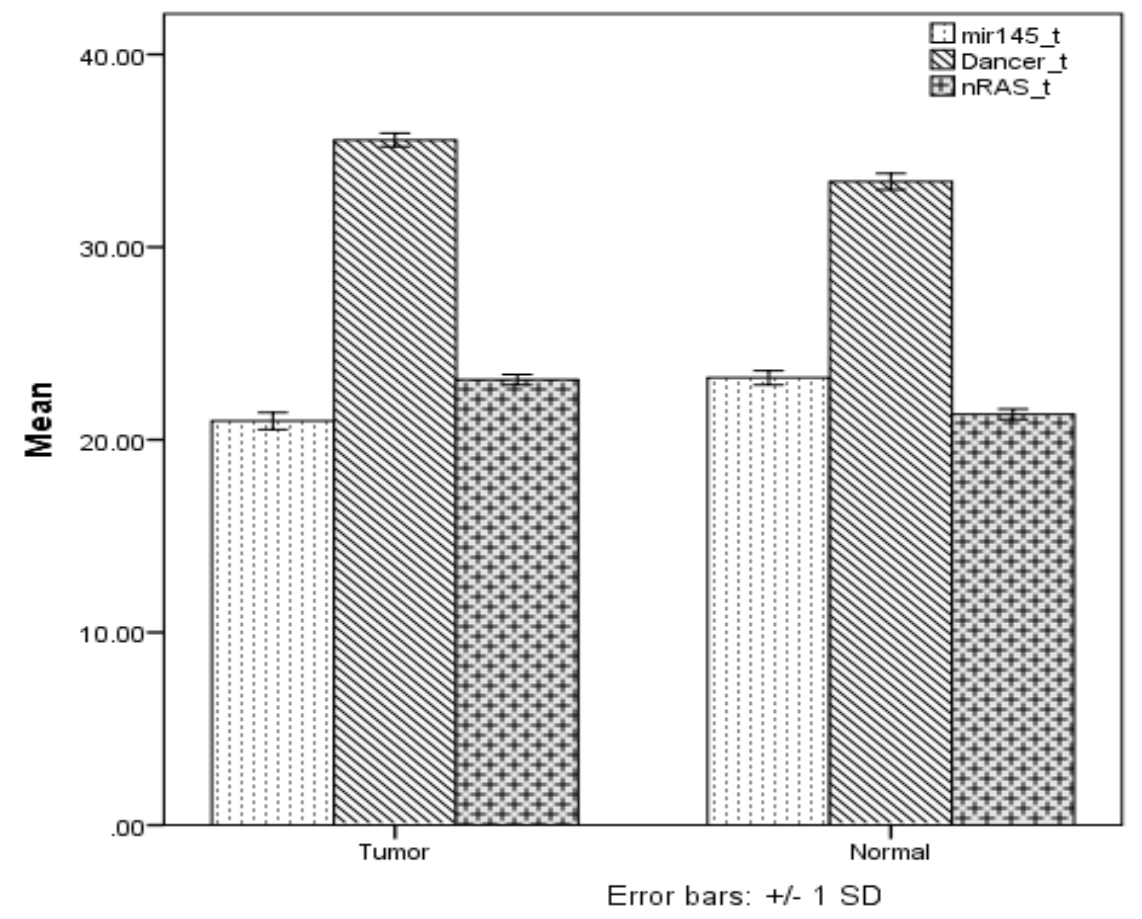

Figure 2. Mean \pm SD of Expression Level of miR145-5p, DANCR and nRAS in Tumor and Control Groups.

$95 \% \mathrm{CI}=29.322-37.58$ months; $\mathrm{P}=0.001$, Figure 1-A).

Figure 1-B shows that the mean survival time of patients with high- and low- DANCR expression levels was not significantly different $(28.05 \pm 2.28,95 \%$

Table 2. Associations of the miR-145-5p, DANCR and NRAS Expression Levels with the Demographic and Clinical Features of Patients with Colorectal Cancer

\begin{tabular}{|c|c|c|c|c|c|c|}
\hline \multirow[b]{2}{*}{ Characteristics } & \multicolumn{2}{|c|}{ miR-145-5p } & \multicolumn{2}{|c|}{ DANCR } & \multicolumn{2}{|c|}{ NRAS } \\
\hline & Mean \pm SD & p-value & Mean \pm SD & $\mathrm{p}$-value & Mean \pm SD & p-value \\
\hline \multicolumn{7}{|l|}{ Sex } \\
\hline Female & $21.72 \pm 2.21$ & $0.238^{*}$ & $33.12 \pm 3.05$ & $0.354 *$ & $22.04 \pm 1.73$ & $0.121 *$ \\
\hline Male & $20.52 \pm 3.11$ & & $33.73 \pm 2.69$ & & $22.66 \pm 1.71$ & \\
\hline \multicolumn{7}{|l|}{ Age } \\
\hline$\leq 60$ & $21.48 \pm 2.59$ & $0.403 *$ & $34.36 \pm 2.53$ & $0.026^{*}$ & $22.82 \pm 1.69$ & $0.106^{*}$ \\
\hline$>60$ & $20.63 \pm 2.99$ & & $32.93 \pm 2.89$ & & $22.18 \pm 1.74$ & \\
\hline \multicolumn{7}{|l|}{ BMI } \\
\hline$<25$ & $22.08 \pm 1.74$ & $0.013^{*}$ & $33.05 \pm 2.45$ & $0.180^{*}$ & $22.45 \pm 1.85$ & $0.941 *$ \\
\hline$\geq 25$ & $19.74 \pm 3.32$ & & $33.91 \pm 3.11$ & & $22.42 \pm 1.65$ & \\
\hline \multicolumn{7}{|l|}{ Grade } \\
\hline Grade I & $23.26 \pm 0.96$ & $0.113 * *$ & $33.19 \pm 2.50$ & $0.273 * *$ & $22.50 \pm 1.58$ & $0.656^{* *}$ \\
\hline Grade II & $20.90 \pm 2.85$ & & $33.83 \pm 2.93$ & & $22.50 \pm 1.84$ & \\
\hline Grade III+IV & $19.89 \pm 2.95$ & & $32.34 \pm 2.83$ & & $21.96 \pm 1.45$ & \\
\hline \multicolumn{7}{|l|}{ Tumor size $(\mathrm{cm})$ : } \\
\hline$\leq 5$ & $23.26 \pm 2.65$ & $0.875^{*}$ & $32.3 \pm 2.99$ & $0.119 *$ & $21.57 \pm 1.54$ & $0.416^{*}$ \\
\hline$>5$ & $23.15 \pm 1.87$ & & $33.76 \pm 2.68$ & & $21.98 \pm 1.61$ & \\
\hline \multicolumn{7}{|l|}{ Stage } \\
\hline Stage I & $21.13 \pm 3.01$ & $0.025 * *$ & $32.32 \pm 2.79$ & $0.442 * *$ & $21.60 \pm 1.56$ & $0.256^{* *}$ \\
\hline Stage II & $22.09 \pm 2.06$ & & $33.74 \pm 2.75$ & & $22.36 \pm 1.74$ & \\
\hline Stage III+ IV & $19.82 \pm 3.14$ & & $33.52 \pm 2.92$ & & $22.69 \pm 1.74$ & \\
\hline \multicolumn{7}{|l|}{ Smoking } \\
\hline Never+ Occasionally & $21.07 \pm 2.92$ & $0.347 *$ & $34.81 \pm 2.83$ & $0.117^{*}$ & $23.83 \pm 1.29$ & $0.006^{*}$ \\
\hline Current & $20.87 \pm 2.23$ & & $33.31 \pm 2.80$ & & $22.23 \pm 1.70$ & \\
\hline
\end{tabular}

*, Significant level is under independent-t-test; **, significant level is under analysis of variance (ANOVA) test. 
Table 3. Univariate and Multivariate Cox Regression Analysis Results for Patients with Colorectal Cancer

\begin{tabular}{lcccc}
\hline & \multicolumn{2}{c}{ Univariate analysis } & \multicolumn{2}{c}{ Multivariate analysis } \\
Characteristics & HR $(95 \%$ CI) & P-value & HR $(95 \%$ CI $)$ & P-value \\
\hline Sex (Male vs. Female*) & $1.316(0.457-3.788)$ & 0.611 & $0.915(0.245-3.425)$ & 0.896 \\
Age (> 60 vs. $\leq 60^{*}$ year) & $0.512(0.190-1.375)$ & 0.184 & $0.415(0.145-1.189)$ & 0.101 \\
BMI (<25* vs. $\geq 25)$ & $1.240(0.461-3.332)$ & 0.67 & $1.678(0.532-5.293)$ & 0.377 \\
Grade (I vs. III+IV*) & $0.706(0.326-3.227)$ & 0.959 & $0.941(0.276-3.209)$ & 0.922 \\
Grade (II vs. III+IV*) & $0.367(0.041-3.290)$ & 0.37 & $0.247(0.024-2.500)$ & 0.236 \\
Tumor size (> 5 vs. $\leq 5^{*}$ cm) & $1.315(0.493-3.506)$ & 0.584 & $2.183(1.403-3.477)$ & 0.039 \\
Stage (II vs. I*) & $1.622(0.199-3.861)$ & 0.651 & $1.560(0.161-5.104)$ & 0.701 \\
Stage (III+ IV vs. I*) & $2.181(1.241-7.854)$ & 0.026 & $2.274(1.218-12.690)$ & 0.034 \\
Smoking (Current vs. Never+ Occasionally*) & $3.144(0.991-9.977)$ & 0.052 & $1.271(0.425-3.560)$ & 0.722 \\
miR-145-5p (low vs. high*) & $6.067(1.716-21.453)$ & 0.005 & $10.759(1.818-63.679)$ & 0.009 \\
DANCR (high vs. low*) & $1.691(0.613-4.666)$ & 0.31 & $1.582(0.495-5.052)$ & 0.439 \\
NRAS (high vs. low*) & $2.614(0.905-7.554)$ & 0.076 & $4.120(1.032-16.448)$ & 0.045 \\
\hline
\end{tabular}

*, The category marked with; “*”, is considered as the baseline level.

$\mathrm{CI}=23.59-32.51$ and $29.20 \pm 2.95,95 \% \mathrm{CI}=23.41-34.99$ months, respectively; $\mathrm{P}=0.302)$. Figure $1-\mathrm{C}$ shows a significantly different survival time $(\mathrm{P}=0.046)$ between high NRAS $(31.20 \pm 2.48,95 \% \mathrm{CI}=26.33-36.07)$ versus low NRAS expression levels $(26.05 \pm 2.65,95 \%$ $\mathrm{CI}=20.86-31.24)$.

Figure 2 shows the average of expression level of miR145-5p, DANCR and NRAS in tumor and normal tissues.

\section{Discussion}

This study aimed to investigate the expression of miRNA-145-5p, DANCR, and NRAS on the survival rate of patients with colorectal cancer. Determining the role of these markers in the prognosis of patients with colorectal cancer can be important for purposes such as screening, early diagnosis, predicting response to treatment, as well as observing the progression of the disease.

An important finding in our study was that under the expression of the miRNA-145-5p gene increases the risk of death from CRC. Consistent with previous research, abnormal and limited expression of the miRNA-145-5p leads to overgrowth and motility of the cell and tumorigenesis. Lower expression levels revealed that miRNA-145-5p can be utilized as diagnostic biomarkers in colorectal cancer. This may also introduce this microRNA as CRC therapeutic target (Chen et al., 2020; Vardini et al., 2017; Xu et al., 2019).

We report a significant relationship between overexpression of NRAS and death from colorectal cancer. Activating mutation in members of the RAS oncogene family one of which is NRAS gene has been found in a variety of human malignancies, suggesting a dominant role in carcinogenesis. In colorectal cancer, NRAS mutations are common and contribute to malignant progression (Vaughn et al., 2011; Irahara et al., 2010).

Based on our result, overexpression of DANCR is associated with death from colorectal cancer. Although the results of our study do not bear statistical significance; this may be due to the low sample size. New research has identified the role of DANCR in tumor progression. Normally, DANCR acts as an oncogenic factor by promoting cancer cell proliferation, invasion, metastasis, chemo-resistance, epithelial-mesenchymal transition (EMT), cancer-stemness features, and angiogenesis (Yan et al., 2020).

The present study indicated that men had the highest mortality rate compared to women. This increased vulnerability of men to death from colorectal cancer may be due to several biological and gender-related (behavioral) factors. Men are more likely to have a diet high in red and processed meat, be the heavy consumer of alcohol, and to smoke. Men also have a greater propensity to deposit visceral fat which is associated with an increased risk of colorectal cancer. On the other hand, lower risk of mortality from CRC in women can be attributed to the protective effect of the estrogen hormone. Recent advances in the molecular biology of colorectal cancer indicated that estrogen may preferentially protect against microsatellite unstable cancers through its effect on selected molecular targets. On the other hand, over the past three decades, epidemiological studies in women have consistently shown that an increase in female hormones such as estrogen and progestin as a result of pregnancy or use of exogenous steroid hormones is associated with a lower risk for developing colorectal cancer (White et al., 2018; Brenner et al., 2007; Lin and Giovannucci, 2010).

Our finding revealed that the body mass index equal to or greater than $25 \mathrm{~kg} / \mathrm{m}^{2}$, increases the risk of death from colorectal cancer. Numerous findings suggest that obesity, especially central fat, increases the risk of colorectal cancer by increasing inflammatory responses, developing metabolic syndrome, and increasing insulin resistance (Donohoe et al., 2010; O’Sullivan et al., 2018).

The risk of death from colorectal cancer increases with the growing tumor size and grade of disease. This matter can be attributed to further invasion of the disease in the end stages of the disease and tumor metastasis to other parts of the body (Kolligs, 2016). Another interesting finding in our study was that smoking increases the risk 
of death from colorectal cancer. Numerous studies in North America show that $15-20 \%$ of colorectal cancers can be attributed to smoking. A possible explanation is that smoking could cause intestinal polyps and more aggressive tumors. Although the direct association between smoking and death from colorectal cancer could be due to different behavioral correlates of smoking, i.e., the tendency for smokers to delay seeking medical care (Alikhani et al., 2009; Botteri et al., 2008). Of course, the limitations of this study should not be overlooked. First, the limitation was the small sample size, which we have tried to discuss cautiously. Second, we merged some categories of independent variables and then analyzed them due to the low-frequency of samples in some categories (e.g., grade and stage of the disease).

In conclusion, our findings demonstrated that the expression levels of NRAS and DANCR in the patients with colorectal cancer were higher than that of the control group. On the other hand, miRNA-145-5p expression was lower in cases than in the control group. These expression levels revealed that miRNA-145-5p and NRAS can be utilized as diagnostic biomarkers in colorectal cancer. This may also introduce the microRNA as colorectal cancer therapeutic targets.

\section{Abbreviations}

CRC, Colorectal Cancer; miRNAs, MicroRNAs; LncRNAs, Long noncoding RNAs; DANCR, Differentiation Antagonizing Non-Protein Coding RNA; CI, confidence interval; HR, Hazard ratio.

\section{Author Contribution Statement}

Fatemeh Baahreini: Conceptualization, Methodology, Software, Formal analysis, Writing-original draft, Writing-review \& editing. Masoud Saeidijam: Writing-review \& editing. Saeid Afshar: Software, Formal analysis Writing-review \& editing. Zahra Mousivand: Software, Formal analysis, Review. Rezvan Najafi: Conceptualization, Review \& editing.

\section{Acknowledgments}

This study was supported by Vic-chancellor for Research and Technology of Hamadan University of Medical Sciences (No. 9705162891).

Ethical Approval

Ethical issues (Including plagiarism, informed consent, misconduct, data fabrication and/or falsification, double publication and/or submission, redundancy, etc.) have been completely observed by the authors. The ethical protocol of this study was approved by the Ethics Committee of Hamadan University of Medical Sciences. (Ethical code: IR.UMSHA.REC.1397.310).

\section{Availability of data}

Data can be obtained from the corresponding author, Dr. Fatemeh Bahreini, Assistant Professor, Department of Molecular Medicine \& Genetics, Hamadan University of Medical Sciences, Hamadan, Iran. e-mail address: f.bahreini2824@gmail.com

\section{Conflict of interest}

The authors declared no potential conflicts of interest.

\section{References}

Aguiar Junior S, Oliveira M, Silva D, et al ( 2020). Survival of patients with colorectal cancer in a cancer center. Arq Gastroenterol, 57, 172-7.

Alikhani S, Delavari A, Alaedini F, et al (2009). A provincebased surveillance system for the risk factors of noncommunicable diseases: A prototype for integration of risk factor surveillance into primary healthcare systems of developing countries. Public Health, 123, 358-64.

Botteri E, Iodice S, Bagnardi V, et al (2008). Smoking and colorectal cancer: a meta-analysis. JAMA, 300, 2765-78.

Brenner H, Hoffmeister M, Arndt V, et al (2007). Gender differences in colorectal cancer: implications for age at initiation of screening. Br J Cancer, 96, 828-31.

Chen Q, Zhou L, Ye X, et al (2020). miR-145-5p suppresses proliferation, metastasis and EMT of colorectal cancer by targeting CDCA3. Pathol Res Pract, 216, 152872.

Cheng V, Wong S, Chuang V, et al (2020). The role of community-wide wearing of face mask for control of coronavirus disease 2019 (COVID-19) epidemic due to SARS-CoV-2. $J$ Infect, 81, 107-14.

Donohoe C, Pidgeon G, Lysaght J, et al (2010). Obesity and gastrointestinal cancer. Br J Surg, 97, 628-42.

GBD Colorectal Cancer Collabrators (2019). The global, regional, and national burden of colorectal cancer and its attributable risk factors in 195 countries and territories, 19902017: a systematic analysis for the Global Burden of Disease Study 2017. Lancet Gastroenterol Hepatol, 4, 913-933.

Irahara N, Baba Y, Nosho K, et al (2010). NRAS mutations are rare in colorectal cancer. Diagn Mol Pathol Am J Surg Pathol Part B, 19, 157.

Kolligs FT (2016). Diagnostics and epidemiology of colorectal cancer. Visceral Med, 32, 158-64.

Lee CH, Chang SC, Tung HY, et al (2018). The risk factors affecting survival in colorectal cancer in Taiwan. Iran $J$ Public Health, 47, 519-30.

Lin JH, Giovannucci E (2010). Sex hormones and colorectal cancer: what have we learned so far? Oxford University Press.

Maajani K, Khodadost M, Fattahi A, et al (2019). Survival rate of colorectal cancer in Iran: a systematic reviewe and metaanalysis. Asian Pac J Cancer Prev, 20, 13-21.

Osullivan J, Lysaght J, Donohoe CL, et al (2018). Obesity and gastrointestinal cancer: the interrelationship of adipose and tumour microenvironments. Nat Rev Gastroenterol Hepatol, 15, 699-714.

Rawla P, Sunkara T, Barsouk A (2019). Epidemiology of colorectal cancer: incidence, mortality, survival, and risk factors. Gastroenterology, 13, 89-103.

Taheri Soodejani M, Mirzaei H, Mahmoodi Manesh M, et al (2019). Incidence of colorectal cancer and adenomatous polyps after a two-step screening in Isfahan Province, Iran in 2018. J Gastrointestinal Cancer, 51, 850-4.

Vardini S, Baradaran B, Golestania-Eimani B (2017). Evaluation of miR-143 and miR-145 expression in tumors and tumor margins in patients with colorectal cancer. $J$ Isfahan Med Sch, 35, 1504-8. 
Vaughn CP, Zobell SD, Furtado LV, et al (2011). Frequency of KRAS, BRAF, and NRAS mutations in colorectal cancer. Genes Chromosomes Cancer, 50, 307-12.

White A, Ironmonger L, Steele RJ, et al (2018). A review of sexrelated differences in colorectal cancer incidence, screening uptake, routes to diagnosis, cancer stage and survival in the UK. BMC Cancer, 18, 1-11.

Xu L, Zhang Y, Tang J, et al (2019). The Prognostic Value and Regulatory Mechanisms of microRNA-145 in Various Tumors: A Systematic Review and Meta-analysis of 50 Studies. Cancer Epidemiol Biomarkers Prev, 28, 867-81.

Yan Y, Shi Q, Yuan X, et al (2020). DANCR: an emerging therapeutic target for cancer. Am J Transl Res, 12, 4031.

\section{c) (i) (8)}

This work is licensed under a Creative Commons AttributionNon Commercial 4.0 International License. 